\title{
Measuring Sound Detection and Reaction Time in Infant and Toddler Cochlear Implant Recipients Using an Observer-Based Procedure: A First Report
}

\author{
Vasant K. Dasika, ${ }^{1}$ Lynne A. Werner, ${ }^{2}$ Susan J. Norton, ${ }^{3,1}$ Kaibao Nie, $^{1}$ and Jay T. Rubinstein ${ }^{1,3}$
}

\begin{abstract}
Objectives: First, to establish the feasibility of the observer-based psychophysical procedure (OPP) in measuring sound detection in infant and toddler cochlear implant $(\mathrm{Cl})$ recipients. Second, to measure the psychometric function for detection (PFD) from individual subjects. Third, to determine whether reaction time (RT) provides information about the auditory sensitivity of young $\mathrm{Cl}$ users.
\end{abstract}

Design: Twelve $\mathrm{Cl}$ recipients, 11 to 32 mo old, participated in our study. Initially, tones were presented in sound field, and children learned to respond when they heard tones but not at other times. Once an $80 \%$ correct criterion was met in sound field, a novel stimulation paradigm was used to present stimuli to a single electrode while the child listened to acoustic input on most other electrodes using their usual map. The PFD and RT were measured using this single-electrode stimulation paradigm.

Results: Eleven subjects met criterion, 6 within the minimum possible number of trials. For eight subjects, the asymptotic level of detecting single-electrode stimuli averaged $86 \%$ correct, similar to levels achieved by normal-hearing infants and toddlers detecting pure tones. The PFD slope of infant and toddler $\mathrm{Cl}$ recipients was less than or equal to the slope for adult $\mathrm{Cl}$ users reported in previous studies. RT decreased significantly with stimulus level in four children.

Conclusions: These preliminary results suggest that psychophysical detection data can be obtained from infant and toddler $\mathrm{Cl}$ recipients using OPP. The PFD of young $\mathrm{Cl}$ users may be shallower than that of adult $\mathrm{Cl}$ users. Relatively good asymptotic detection performance implies that young $\mathrm{Cl}$ users are more attentive to sound than has been suggested in previous studies. RT tended to be a less reliable measure of detection, but methodological changes could improve its utility.

(Ear \& Hearing 2009;30;250-261)

\section{INTRODUCTION}

Cochlear implantation is the standard of care for treating deafness in infants and young toddlers. Although electric hearing (EH) children develop functional levels of spoken language comprehension and speech production, they are still hearing impaired and exhibit degraded speech reception, vocabulary, music reception, and even fine motor skills, compared with normal-hearing (NH) children (e.g., Eisenberg et al. 2006; Gfeller et al. 1999; Horn et al. 2006; Mukari et al. 2007; Nakata et al. 2005; Olszewski et al. 2006; Stordahl 2002; Uziel et al. 2007; Vongpaisal et al. 2006). Additional information about hearing development in infant and toddler cochlear

\footnotetext{
${ }^{1}$ Department of Otolaryngology, Virginia Merrill Bloedel Hearing Research Center, University of Washington; ${ }^{2}$ Department of Speech and Hearing Sciences, University of Washington; and ${ }^{3}$ Divisions of Audiology and Otolaryngology, Seattle Children's, Seattle, WA.
}

implant (CI) users is required, and behavioral data are considered the "gold standard" for assessing hearing.

The observer-based psychophysical procedure (OPP), developed by Werner et al. is an established method for obtaining behavioral data from NH infants (e.g., Olsho et al. 1987; Werner 1995). In OPP, a trained observer, blinded to trial type, judges whether or not a signal was presented on a trial based on the infant's behavior. When signal trials are correctly detected, the infant is rewarded by the activation of a "reinforcing" mechanical toy or video program. If the observer can distinguish signal from no-signal trials in this way, it is clear that the infant heard and responded to the signal. OPP has been used to assess many psychoacoustical abilities, including detection, discrimination, and categorization (e.g., Marean et al. 1992; Werner \& Boike 2001). In addition, OPP has been adapted recently to measure sound localization in EH toddlers (GriecoCalub et al. 2008).

Although several methods are available to test EH infants and toddlers, OPP seems especially well suited to obtaining psychophysical data from this population. One of the first methods used, the visual habituation procedure teaches infants to fixate on a visual pattern to trigger the presentation of a particular sound (e.g., Houston et al. 2003a; Miyamoto et al. 2005). A second method, the preferential looking procedure, measures the length of time that an infant looks at one of two video events, displayed side-by-side, while a sound that matches one of the events is played (e.g., Barker \& Tomblin 2004; Houston et al. 2003b). In either procedure, an infant who is not interested in the sound may not look at the visual display. In a third method, the conditioned head-turn procedure, infants learn to turn their head toward the reinforcer when they hear a sound or a change in a sound (e.g., Eisenberg et al. 2005, 2007; reviewed by Werker et al. 1997). The appeal of the reinforcer, rather than that of the target sound, largely determines whether the infant will respond in this procedure. In addition, the conditioned head-turn procedure is well suited to measuring sensitivity when multiple stimulus values must be assessed (e.g., to establish threshold), whereas visual habituation and preferential looking procedures are not. OPP has the same advantages as the conditioned head-turn procedure, but in addition, it permits any observable response, including a head turn, to be used by the observer in correctly identifying signal trials. OPP was initially developed to test $\mathrm{NH}$ infants younger than 6 mo old who do not produce reliable head turns (Olsho et al. 1987). This flexibility could be helpful in testing hearing-impaired infants who may not have the same responses to sound as NH children of the same age.

The first objective of this study was to establish the feasibility of using OPP with infant and toddler CI recipients. 
TABLE 1. Subject demographics, device details, and any medical or procedural issues

\begin{tabular}{|c|c|c|c|c|c|c|c|c|c|c|}
\hline Subject & Gender & Ear & $\begin{array}{c}\text { Age at } \\
\text { implantation } \\
\text { (mo) }\end{array}$ & $\begin{array}{c}\text { Age at } \\
\text { first } \\
\text { visit (mo) }\end{array}$ & Implant & Strategy & $\begin{array}{l}\text { Pulse rate } \\
\text { per channel } \\
\text { (pulses/sec) }\end{array}$ & $\begin{array}{l}\text { Pulse } \\
\text { width } \\
(\mu \mathrm{sec})\end{array}$ & $\begin{array}{l}\text { Stimulating } \\
\text { electrode }\end{array}$ & $\begin{array}{l}\text { Special } \\
\text { issues }\end{array}$ \\
\hline s3 & M & $\mathrm{R}$ & 12 & 14 & Freedom & ACE (RE) & 1800 & 20 & E15, E8† & - \\
\hline s4 & $\mathrm{F}$ & $\mathrm{L}$ & 14 & 21 & HiRes 90K & HiRes-P & 5156 & 10.8 & $\mathrm{E} 7, \dagger \mathrm{E} 3$ & Microcephaly \\
\hline s5 & M & $\mathrm{R}$ & 18 & 32 & Freedom & ACE (RE) & 1800 & 20 & E16,† E11 & $\begin{array}{l}\text { Used OPP + } \\
\text { VROCA }\end{array}$ \\
\hline s6 & M & B & 10 & 14 & $\begin{array}{l}\text { MED-EL } \\
\quad \text { PulsarCl }\end{array}$ & $\mathrm{CIS}+$ & 1515.2 & 24.2 & Not tested & $\begin{array}{l}\text { Implant-in-a-box } \\
\text { unavailable }\end{array}$ \\
\hline s12 & M & $\mathrm{L}$ & 20 & 27 & HiRes $90 \mathrm{~K}$ & $\begin{array}{l}\text { HiRes-P } \\
\quad \text { w/Fidelity } 120\end{array}$ & 3712 & 18 & E7 & Trisomy 9p \\
\hline
\end{tabular}

* Subject was remapped across multiple visits.

† Did not see any consistent response to specified electrode(s).

$\neq s 9$ is a bilateral recipient; only the $R$ ear was tested.

Sound detection was the first capacity examined because it is the most basic auditory ability and is essential for all other aspects of hearing. The second objective of this study was to measure the psychometric function for detection (PFD) in $\mathrm{EH}$ infants and toddlers using OPP. Information about the upper asymptote and slope of the PFD may provide information about the underlying detection process, including auditory attention, and could also inform the development of adaptive testing methods. In addition, the threshold derived from the PFD could better define the clinically programmed, minimal-level of stimulation current elicited on one electrode, that is, the T-level. The PFD has been described for NH infants, children, and adults (e.g., Allen \& Wightman 1994; Bargones et al. 1995; Olsho et al. 1987; Schneider et al. 1989; Trehub et al. 1980; Werner \& Boike 2001), hearing-impaired adults (e.g., Carlyon et al. 1990), and EH adults (Donaldson et al. 1997; Donaldson \& Viemeister Reference Note 1). However, to our knowledge, it has not yet been measured in EH infants and toddlers.

The dependence of reaction time (RT) on stimulus level was also examined to see whether information would be provided about the detection process. RT strongly decreases with stimulus intensity at near-threshold levels in NH adults (e.g., Epstein \& Florentine 2006; Heil et al. 2006; Pfingst et al. 1975). Similarly, at suprathreshold levels, RT decreases with stimulus intensity in both NH infants (Leibold \& Werner 2002) and adults (e.g., Humes \& Ahlstrom 1984; Luce 1991). In implanted animals, RT decreases as the electrical charge delivered increases (e.g., monkey: Pfingst 1984; cat: Walloch et al. 1980). Two studies measuring closed-set speech-identification-in-competing-noise in $\mathrm{NH}$ and $\mathrm{EH}$ children suggest that RT can provide more information than response accuracy alone in certain stimulus conditions (Eisenberg et al. 2005; Johnson et al. Reference Note 2). In these studies, RT decreased monotonically as the speech-to-competitor level increased from -10 to 0 to $+10 \mathrm{~dB}$, whereas response accuracy remained at ceiling performance $(100 \%$ correct $)$. For these reasons, RT information was collected as part of the PFD measurement. We generally hypothesized that RT would decrease with stimulus level, whereas detection accuracy would increase.

\section{MATERIALS AND METHODS}

\section{Subjects}

Twelve subjects were tested. For each subject, Table 1 lists basic demographic and implant-related characteristics and any unique medical or experimental issues. Subject age at the time of implantation ranged from 9 to $20 \mathrm{mo}$, as listed in the fourth column of Table 1. Subject age on the date of the first visit ranged from 11 to $32 \mathrm{mo}$, with a median age of $18.5 \mathrm{mo}$, as listed in the fifth column of Table 1. EH experience ranged from 1.5 to $14 \mathrm{mo}$ at the time of the first visit. Subjects used Cochlear $^{\mathrm{TM}}$, Advanced Bionics ${ }^{\circledR}$ (AB) or MED-EL devices, as listed in the sixth column of Table 1. Not all data were obtained from all subjects, as detailed in the sections that follow. For example, for the single MED-EL subject s6, PFD and RT data were not collected because an "implant-in-a-box" was unavailable to verify the stimuli, as noted in the last column of Table 1. All subjects received audiologic care at Seattle Children's in Seattle, WA, where clinical mapping information was obtained for each subject. All procedures were approved by the Institutional Review Board at Seattle Children's and the University of Washington.

\section{Stimuli}

Two types of stimuli were used in this experiment: acoustic tone bursts presented in sound field and trains of current pulses delivered to a single electrode in the implanted array. Pilot testing indicated that children completed more trials and 
produced more reliable conditioned responses to tones in sound field than to standard single-electrode stimulation. Consequently, sound field stimuli were used during the initial training phases of the experiment while the child listened through his or her usual speech processor program (map). After training criterion was reached, a new method for delivering single-electrode stimuli with ambient sound processing was used to measure the PFD and RT.

Sound field stimuli were five identical $300 \mathrm{msec}, 10 \mathrm{msec}$ rise/fall, pure-tone bursts (in sine phase), with $300 \mathrm{msec}$ silent periods between bursts, delivered from a speaker located approximately $45^{\circ}$ to the child's right $1.5 \mathrm{~m}$ away. Steady state burst level was fixed at $60 \mathrm{~dB}$ SPL for AB and MED-EL users and $65 \mathrm{~dB}$ SPL for Cochlear users. These values are based on the default settings that map SPL to comfort level, the clinically specified maximum stimulation current level for a given electrode defined as the $\mathrm{C}$ - or M-level for the Cochlear or $\mathrm{AB}$ device respectively. Tone frequency matched the center frequency $(\mathrm{CF})$ of the filter in the CI recipient's array closest to $1000 \mathrm{~Hz}$, for example, $1000 \mathrm{~Hz}$ for Cochlear, $1076 \mathrm{~Hz}$ for AB, and $1062 \mathrm{~Hz}$ for MED-EL devices. These stimuli seemed to be clearly audible to all subjects.

A stimulation paradigm referred to as "Live Single Electrode Stimulation" (LSES) was developed to deliver currentpulse trains to a single-electrode while ambient sounds were concurrently processed during testing. In LSES, ambient sounds were processed through a device-specific speech processor and single-electrode stimuli were presented by delivering the tone burst stimuli to the auxiliary input jack of the speech processor. Only electrodes affected by the tone burst stimuli were modified; other electrodes were not changed. LSES allowed children to hear the reinforcer toys, the parent, and the assistant during testing.

Before the session, stimuli were verified using a devicespecific implant-in-a-box with $5-\mathrm{k} \Omega$ load resistors to ensure that the desired pulse-train patterns occurred on the target electrode with no stimulation on any other electrodes. To compare measured PFD with clinical parameters, it was necessary to determine the current level at clinical parameter levels. Sometimes, the expected output current given by manufacturer-provided equations differed from implant-in-abox measurements. To provide comparable measures of the current levels arising from stimulation at clinical parameter levels, the clinical fitting software was used to generate clinical threshold or comfort level pulses, whereas the output was measured using the implant-in-a-box. The same implant-in-abox was used for all measurements for each type of device to reduce the potential effects of device-to-device variability. Each device-specific implant-in-a-box was not explicitly calibrated. However, as long as any differences in current produced by the implant-in-a-box and the subject's internal device were constant across level, relative measures such as PFD slope, RT slope, or the PFD threshold in relation to clinical parameters would not be affected.

Only sound field data were collected for the MED-EL user s6 because an appropriate implant-in-a-box was unavailable. LSES procedures for the other two device types are given in the following paragraphs.

Cochlear Nucleus Freedom Implant LSES Settings • Before the session, the Custom Sound clinical fitting software was used to program a laboratory Nucleus Freedom sound proces- sor* so that slot P1 held the child's latest clinical map, and slots P2, P3, and P4 held up to three different custom maps. Each custom map allowed testing of a different electrode. For custom maps, the accessory mixing ratio was set to $10: 1$ (adjusted from the typical default of $3: 1$ ) to ensure that no activity on any electrodes occurred in a quiet sound booth and that activity occurred in response to audible sound. Because of spectral overlap between adjacent filters, a minimum of two electrodes immediately adjacent to the electrode of interest were turned off by setting the C-level to 1 (the minimum nonzero value) and the T-level to 0 . T-level at the electrode of interest was also set to 0 . Most other clinical map parameters were left unchanged (see Map Details). In this situation, when an unmodulated tone with a frequency matching the estimated $\mathrm{CF}$ of the electrode of interest was presented to the external accessory input of the Freedom controller/processor that was in external accessory (EA) mode, unmodulated pulses were produced only on that electrode. Pulse amplitude could be varied progressively from 0 to $\mathrm{C}$-level by increasing the amplitude of the tone. This is currently the most reliable method for presenting single-electrode stimuli with concurrent live audio processing in the Cochlear Freedom device.

AB Clarion HiRes 90K Implant LSES Settings • A laboratory Platinum Series Body-Worn sound processor was programmed using the SoundWave clinical fitting software; P1 held the clinical map, and slots P2 and P3 held two custom maps. Again, T-level was set to 0 on the electrode of interest, and a minimum of two adjacent electrodes were turned off (M-level set to 1 , T-level set to 0 ). In addition, the input dynamic range was reduced from the typical clinical map values of 60 to $70 \mathrm{~dB}$ to 40 $\mathrm{dB}$. The reduction in input dynamic range ensured that no output occurred on any electrode in a quiet sound booth. The audio mixing ratio was left at the default 50/50.

Map details - Aside from the changes made to the child's latest clinical map specified in Cochlear Nucleus Freedom Implant LSES Settings and AB Clarion HiRes 90K Implant LSES Settings, most other parameters, including the processing strategy, pulse rate, pulse width, stimulation mode, frequency table (Cochlear), and the extended low-frequency default filter setting $(\mathrm{AB})$, were not changed for LSES. The stimulation mode was always monopolar, consistent with current clinical strategies. Processing strategy, pulse rate, and pulse width information are listed in the seventh, eighth, and ninth columns of Table 1. To avoid cuing the observer, assistant, parent, and child on signal presentations, the sound-indicator light on the processor, which by default is on for pediatric users, was either turned off or covered with opaque tape.

Target electrode $\bullet$ For both Cochlear and $\mathrm{AB}$ devices, the stimuli on the target electrode (i.e., the signal that the child listens for) consisted of five current-pulse trains, $300 \mathrm{msec}$ in duration, separated by $300 \mathrm{msec}$, as a consequence of the tone burst stimuli applied to the speech processor. The target electrode was specified by choosing the appropriate custom speech-processor map, and the tone frequency was specified to match the CF of the target electrode.

For Cochlear users, tone frequency was the arithmetic mean of the lower and upper cutoff frequencies correspond-

\footnotetext{
*Both BTE and Bodyworn controllers were used at different phases of the experiment. No differences in single-electrode stimuli were observed between the two controllers.
} 
ing to the target electrode. For example, to stimulate E16 on the Freedom implant with default frequency table settings (Frequency Table no. 22), having a lower cutoff frequency of $938 \mathrm{~Hz}$ and an upper cutoff frequency of $1063 \mathrm{~Hz}$, the tone frequency was $1000 \mathrm{~Hz}$. The resulting current-pulse trains had a delay of $5 \mathrm{msec}$ and a $15 \mathrm{msec}$ linear rise/fall ramp, arising from a combination of the rise fall time on the tone bursts and the automatic gain control of the Freedom processor. Otherwise, pulses were unmodulated in a train and matched those resulting from the "behavioral-measurement mode" of the Custom Sound clinical software.

For $\mathrm{AB}$ users, the tone frequency equaled the $\mathrm{CF}$ of the filter for the given electrode specified by the clinical map. For the more apical electrodes tested, E3 $(\mathrm{CF}=540 \mathrm{~Hz})$ and $\mathrm{E} 7(\mathrm{CF}=1076 \mathrm{~Hz})$, the current-pulse trains were essentially amplitude-modulated by the halfwave rectified tone bursts under the HiRes processing strategy. Modulation resulted from two attributes of the HiRes strategy. First, the effective high cutoff frequency of the low-pass filter after the bandpass-filtering and half-wave rectification stages of processing predicts that a half-wave rectified version of the input tone would modulate the current pulses, provided that the pulse rate is fast enough. Second, pulse rates equaled or exceeded 3480 pulses/sec (Table 1, ninth column), which was more than three times the tone frequency used to test E7 or E3. Consequently, the observed amplitude of the current pulses on these electrodes followed the shape of the half-wave-rectified input tone.

The average current-pulse amplitude during a currentpulse train was used as the measure of stimulus magnitude. Given the half-wave-rectified sinusoidal envelope of the current-pulse trains observed under the HiRes strategy, the average current level was estimated as the average value of a half-wave-rectified sinusoid, or the peak current divided by $\pi$. In contrast, the average current-level per current-pulse train for Cochlear users simply equaled the peak current because pulse trains were unmodulated under the ACE and ACE(RE) strategies.

The stimulating electrode usually had a corresponding filter CF near $1000 \mathrm{~Hz}$ and was typically E16 (CF $=1000$ $\mathrm{Hz})$ for Cochlear users and $\mathrm{E} 7(\mathrm{CF}=1076 \mathrm{~Hz})$ for $\mathrm{AB}$ users, as listed in the 10th column of Table 1. In some cases, testing was attempted with multiple electrodes, as indicated in Table 1. Sometimes no reliable behavioral response was observed from an electrode, and this is indicated by an asterisk in Table 1. For example, E7 was initially stimulated for $\mathrm{s} 4$, but the subject's response was inconsistent. Because s4 had responded well to a $540 \mathrm{~Hz}$ tone in sound field in a previous unpublished study, E3 $(540 \mathrm{~Hz} \mathrm{CF})$ was then stimulated, which elicited consistent response from the subject. Stimulus delivery $\bullet$ For Cochlear users, the tone burst stimuli were delivered to the external accessory socket of the Freedom controller using a Freedom TV Hi/Fi stereo cable (the ring and sleeve contacts of the cable plug were shorted). For AB users, stimuli were delivered to the auxiliary input jack of the body-worn processor using a standard two-conductor cable with a $3.5 \mathrm{~mm}$ mono plug.

\section{General Procedure}

Experiments were conducted in a sound booth. Procedures paralleled those used with NH infants (Werner 1995) with modifications for EH. During sessions, the child was seated on a parent's lap while a trained assistant manipulated quiet toys to keep the child attentive and facing forward. The observer was outside the booth and monitored the child via a one-way glass or a video monitor. The same observer, the first author, conducted all of the sessions. The observer, assistant, and parent all wore headphones. When sound field stimuli were presented, the three adults were presented a masker on every trial to prevent them from hearing the signal. Initially, music was used as a masker, but a more effective narrowband noise centered at the signal frequency was used for most sessions. The observer generally monitored sounds in the booth other than the signal and the assistant monitored audio from the observer. Two stacked smoked-Plexiglas boxes, each containing a mechanical toy, were positioned approximately $45^{\circ}$ to the child's right $1.5 \mathrm{~m}$ away. A video-reinforcer monitor was positioned atop the toy boxes.

OPP is a single interval procedure. The observer began a trial when the subject was quiet and seemed attentive. Either a signal or a no-signal trial was presented randomly under computer control. Trials were $4 \mathrm{sec}$ in duration. The observer knew when a trial started and ended but was blind to the trial type. On a signal trial, the stimulus - sound field tone bursts or LSES current-pulse trains - was immediately presented. On a no-signal trial, no stimulus was presented. The observer's task was to decide whether or not a signal was presented during the trial, based on the child's behavioral response (e.g., head turn toward the reinforcer or shifts gaze). A "Yes" response was entered when the observer pressed a specified key on the computer keyboard. RT was recorded at millisecond resolution and defined as the time of the first "Yes" response that occurred within the trial. If no-key press occurred, a "No" response was registered. Correct answer feedback was given to the observer immediately after a "Yes" response or immediately after the trial if a "No" response was registered. If a "Yes" response occurred during a signal trial (a hit), the child was rewarded immediately by the illumination and activation of one of the two toys or by the display of a video clip for $4 \mathrm{sec}$. In addition to providing a dynamic visual display, the toy or video reinforcer produced audible sound (e.g., drumming and toy motor sounds or the video sound track). If the observer responded "Yes" on a no-signal trial (a false alarm), no reinforcer was presented. Between trials, the assistant brought the child's attention back to center by showing the child quiet toys (e.g., a pinwheel, book, or pompom). The assistant and parent could praise the child after reinforcer activation. The observer could only initiate the next trial after any reinforcer activity had ended.

Three experimental phases were attempted for each child: training, criterion, and testing, paralleling OPP with $\mathrm{NH}$ infants (Werner 1995).

Training and criterion $\bullet$ Sound field stimuli were presented during these phases. The training phase demonstrated the association between the stimulus and the toy/video reinforcer to the child and gave the observer a sense of how a particular 
TABLE 2. Individual subject data showing the number of trials to reach criterion, the total number of visits, hit rate $(H)$, false alarm rate $(F)$, number of trials information (corresponding signal levels are indicated in Fig. 1), correlation between $p(C)_{\max }$ and level, PFD asymptote, PFD slope, $R^{2}$ of 3 -line fit, and RT-versus-level slope

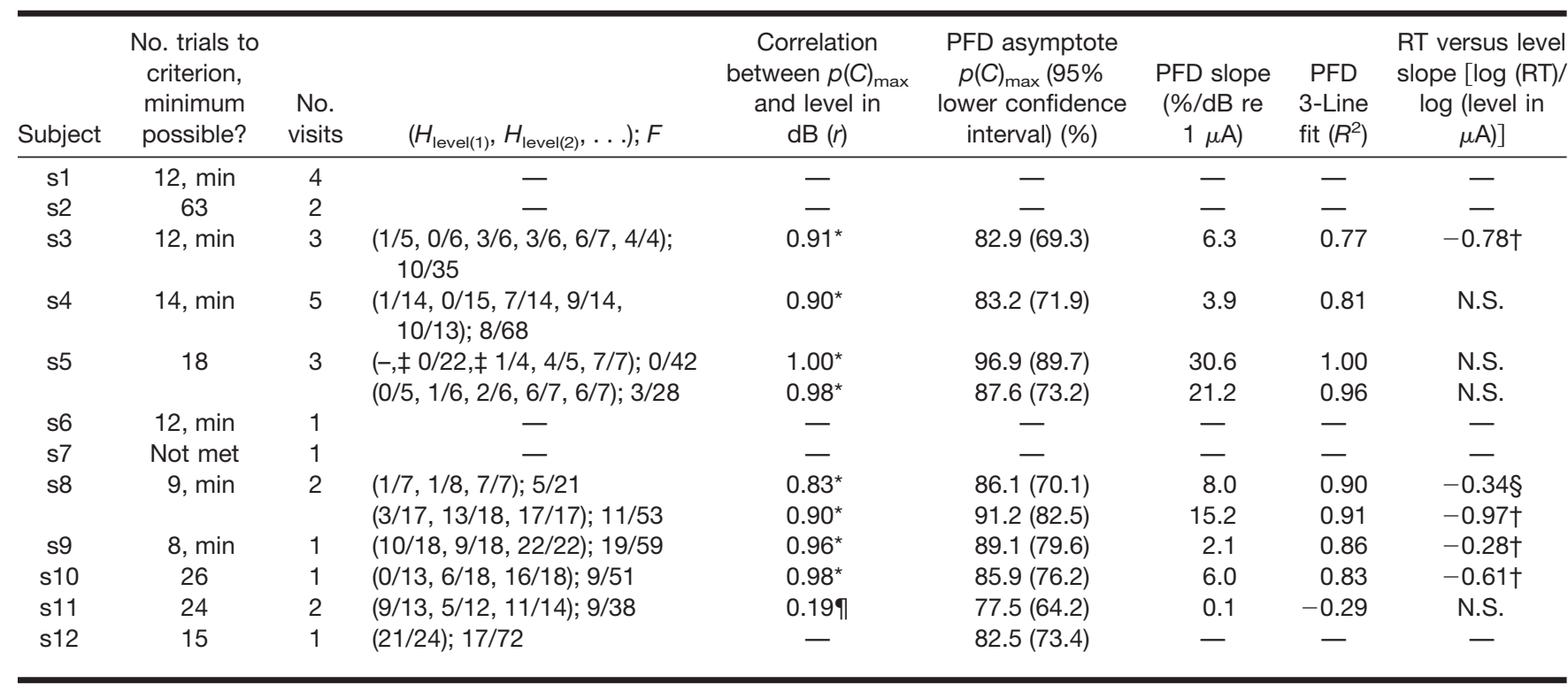

N.S. $p>0.4$.

${ }^{*} p<0.000001$ for a positive relationship.

$+p<0.01$ for a negative relationship.

$¥$ The lower four of the five levels initially presented resulted in no hits; levels were subsequently raised until the PFD was bracketed. For analyses, all lower levels where $H$ was zero were pooled at the highest of such levels. Pooling decreased the positive skew in $p(C)_{\max }$ resulting from the conversion specified in the Materials and Methods section when $\mathrm{H}$ and $\mathrm{F}$ both equal zero and $N_{n s}$ far exceeds $N_{s}$. A reasonable estimate of $p(C)_{\max }$ of $55 \%$ resulted (cf., Fig. 1). Otherwise, $p(C)_{\max }>69 \%$, and performance was statistically on the slope at these levels.

$\S p=0.10$ for a negative relationship.

If $p>0.125$ for a positive relationship.

child responded. Two details were unique to this phase. First, the reinforcer was activated immediately after every signal trial even if the observer did not respond "Yes." Second, 75\% of the trials were signal trials. The training phase continued until the observer was correct on four of the last five trials, with at least one correct rejection. This training phase was typically short, lasting 5 to 10 trials, which is consistent with $\mathrm{NH}$ infant data (Werner 1995). The oldest subject, a 32 mo old child, s5, did not train within 10 trials. For this child, the training phase was repeated and modified so that the assistant could also hear the tones and could model responses for the child. The child subsequently conditioned quickly using a combination of OPP and visually reinforced operant conditioning audiometry. Here, the child dropped a block in a bucket when he heard the signal, a task he was already very familiar with, and the observer used the block dropping to identify signal trials. Strictly speaking, this procedure is not OPP, but the data were similar to those obtained from other children and are therefore included.

The criterion phase generally continued without pause after the training phase. In this phase, the probability of signal and no-signal trials equaled 0.5 with no constraints. The reinforcer was activated only after hits. Trials were presented until the observer correctly identified four of the last five signal trials and four of the last five no-signal trials. The second column of Table 2, which lists individual subject data, indicates the number of trials required to reach criterion for each subject. For some subjects, criterion was met in the minimum possible number of trials given the particular random sequence of signal and no-signal trials presented. The number of trials-to-criterion data from these subjects is followed by the "min" label in Table 2 .

Testing: Measuring the PFD and RT • LSES was used in the testing phase to measure the PFD and RT. No extensive training was required to reestablish children's responses to LSES. A few comfort-level single-electrode trials were generally adequate in subsequent sessions to remind the child of the rules of the game. Like the criterion phase, the reinforcer was activated only after a hit, and signal and no-signal trials were equally likely.

Five signal levels were presented in the early experimental sessions in an attempt to encompass the entire range of the PFD. Based on the variability observed in some of these data, later sessions were conducted using only three levels to increase the number of trials presented per level. The two lower levels attempted to target the slope, and the highest level was set equal to comfort-level to target the asymptote. Signal and no-signal trials were randomly ordered in blocks. For sessions in which five levels were presented, each block was made up of 10 trials, with 1 signal trial at each of five levels and 5 no-signal trials. ${ }^{\dagger}$ For sessions in which three levels were presented, each block was made up of 12 trials, with 2 signal trials at each of three levels and 6 no-signal

\footnotetext{
${ }^{\dagger}$ For s3, seven signal trials were presented at the fifth level. An additional block of randomly ordered no-signal $(N=4)$ and signal $(N=4)$ trials at a sixth (and highest) level was also collected for this subject. Performance at the fifth and sixth levels was similar, so data were pooled to estimate asymptote from a greater number $(N=11)$ of signal trials.
} 
trials. Multiple successive blocks were run during a test session. Werner and Marean (1991) used a similar method of constant stimuli with mixed signal blocks to estimate $\mathrm{NH}$ infant thresholds.

To maintain the child's interest during testing, reinforcement was varied between toy and video, and different toys or video programs could be used. If the child's interest seemed to be fading, the observer could insert a "probe" trial on which the signal was presented at comfort level. Breaks were taken approximately every 10 to $15 \mathrm{~min}$ or as needed, and testing continued until the child became fussy or excessively inattentive or until the scheduled time had elapsed.

The PFD and RT were measured in seven children. Of the other four non-MED-EL users, two subjects (s1 and s2) did not respond reliably at the highest level presented for any of the attempted electrodes, one (s7) did not reach criterion, and one (s12) responded correctly $83 \%$ of the time at the lowest signal levels presented. Thus, for $\mathrm{s} 12$, only asymptotic performance data are reported in response to the highest signal level presented; this signal level equaled $54.8 \mathrm{~dB}$ re $1 \mu \mathrm{A}$.

The PFD and RT described here were obtained in a single visit for each child to reduce the potential influence of day-to-day variability in performance. However, as shown in the third column of Table 2, sometimes more than one visit was necessary to obtain these data. Initially, training and criterion phases were completed in one visit, and testing was completed in a subsequent visit. To reduce the number of visits for subjects after s7, both phases were conducted in the same visit whenever possible, and this was the case for s8, s9, s10, and s12. If the child was not amenable to testing in one visit, an additional visit was scheduled. For example, two additional visits were scheduled for s4 because the first two visits, in which the PFD was attempted to be measured, did not yield usable data. When possible, an additional visit was scheduled to either repeat the PFD measurement or to test a different electrode. Repeated PFD measures were attempted with three subjects (s4, s5, and s8) and obtained from two subjects (s5 and s8). Repeated-measures data are listed in the second rows of the entries for s5 and s8 in Table 2. Experimenter error led to uncertainty about the exact stimulus levels presented to s4 during this subject's last session, precluding inclusion of those data. For s3, the PFD was successfully measured when electrode E15 was stimulated during this subject's second visit. Subsequent testing with E8 was attempted during this subject's final visit. However, $\mathrm{s} 3$ did not produce reliable responses to stimulation of $\mathrm{E} 8$, as indicated in the 10th column of Table 1 .

Signal levels were initially set to span a range of up to approximately $10 \mathrm{~dB}$. Adjustments were made to the levels if needed. Examples of level adjustments made when testing three subjects s4, s5, and s8 are briefly described. Subject s4 was tested for four LSES sessions to test E3. Information obtained during the first two unusable sessions helped to define the levels later presented during the usable session for this subject. For s5, of the five levels initially presented during this subject's first testing visit (or 2nd visit overall), the lower four levels resulted in no hits. Levels were consequently increased until the sloping part of the PFD was targeted. This (final) set of levels was presented to obtain the repeat PFD measurement during s5's final visit. For s8, of the three levels presented during his first visit, postsession analyses revealed that performance was statistically at chance at the lower two levels, whereas performance exceeded $88 \%$ correct at the highest level. The lower two levels were subsequently increased for this subject's second and final visit, which better defined the sloping portion of the PFD for the repeated measure.

Information about the number of signal trials and the number of no-signal trials obtained from each subject is provided in the fourth column of Table 2. These data are given by the denominator of the indicated hit $(H)$ and false alarm $(F)$ proportions. For example, for s3, five trials at the lowest signal level "level(1)", six trials at the second level "level(2)", etc., were presented in addition to the 35 no-signal trials. The resulting hit rate at the lowest signal level was $1 / 5$, or 0.2 , and the false alarm rate was $10 / 35$, or 0.29 .

\section{Data Analyses}

All data from the final set of stimulus levels for each valid testing session, including incomplete trial blocks, were analyzed with the exception of probe trials and trials during which the child's headpiece came off. The number of excluded trials ranged from 0 to 9 across subjects, accounting for 0 to $7 \%$ $(M=3.7 \% ; \mathrm{SD}=3.1 \%)$ of trials completed.

PFD data were plotted in terms of $p(C)_{\max }$, the maximum percent correct that corresponds with a given $d^{\prime}$ (e.g., Bargones et al. 1995; Macmillan \& Creelman 2005). $p(C)_{\max }$ was used to allow comparison with published data from $\mathrm{NH}$ infants and children (Allen \& Wightman 1994; Bargones et al. 1995). To avoid undefined values of $d^{\prime}$, hit rates that equaled 0 or 1 were converted to $0.5 / \mathrm{N}_{\mathrm{s}}$ or $1-0.5 / \mathrm{N}_{\mathrm{s}}$, where $\mathrm{N}_{\mathrm{s}}$ was the number of signal trials presented at a given level. Similarly, false alarm rates that equaled 0 or 1 were converted to $0.5 / \mathrm{N}_{\mathrm{ns}}$ or $1-$ $0.5 / \mathrm{N}_{\mathrm{ns}}$, where $\mathrm{N}_{\mathrm{ns}}$ was the number of no-signal trials. Similar conversions have been used extensively in previous studies (e.g., Bargones et al. 1995; Macmillan \& Creelman 2005). The $95 \%$ confidence intervals for $p(C)_{\max }$ were estimated by generating the sampling distribution of $d^{\prime}$, assuming binomial probabilities of hits and false alarms (cf., Bargones et al. 1995).

\section{RESULTS}

Three questions were addressed in the analyses of the data. First, can EH infants and toddlers be tested with OPP? Second, what are the characteristics of the PFD of EH infants and toddlers? Third, does RT vary systematically with stimulation level in EH infants and toddlers?

\section{Success Rate}

One measure of the utility of a test method is the proportion of subjects who are able to perform the test. Nearly, all of the EH children were successful in OPP in that sense. The criterion level of performance was achieved by 11 of the $12 \mathrm{EH}$ children (92\%) listening through their usual maps in response to 60 to 

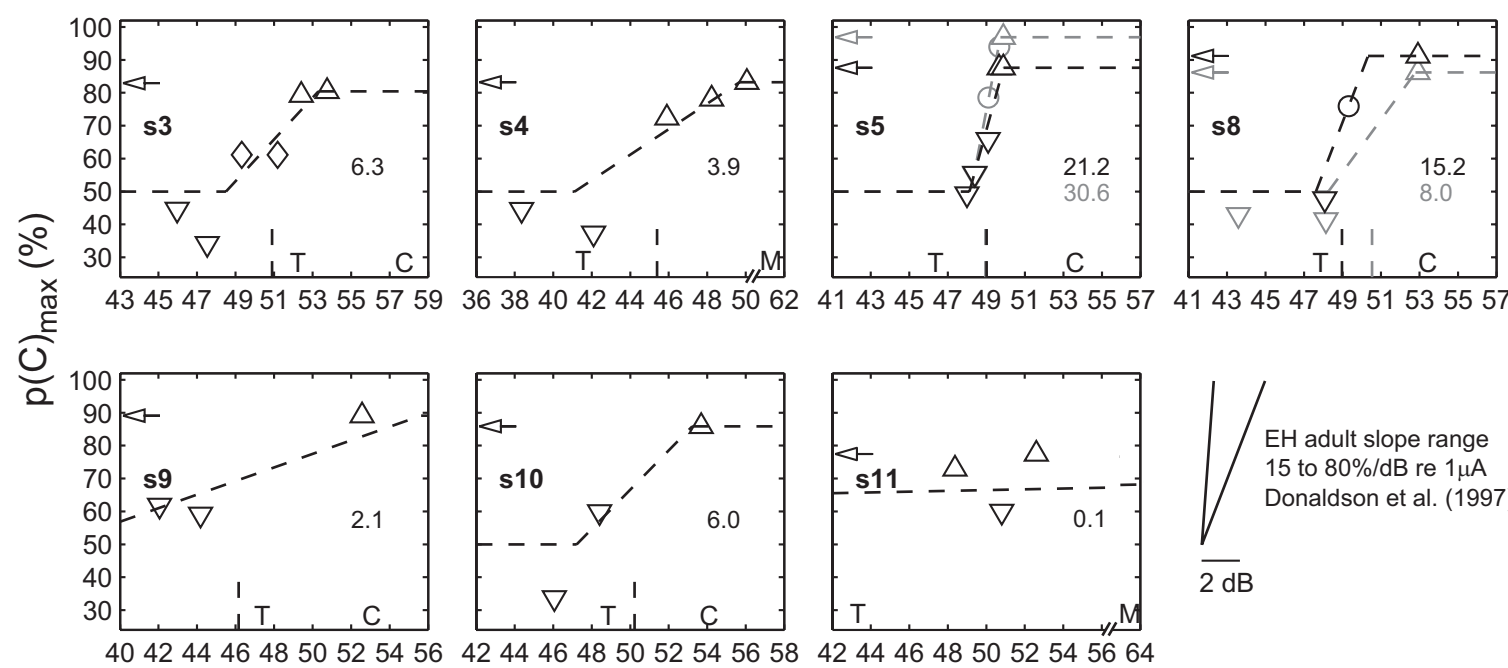

363840424446485062414345474951535557414345474951535557
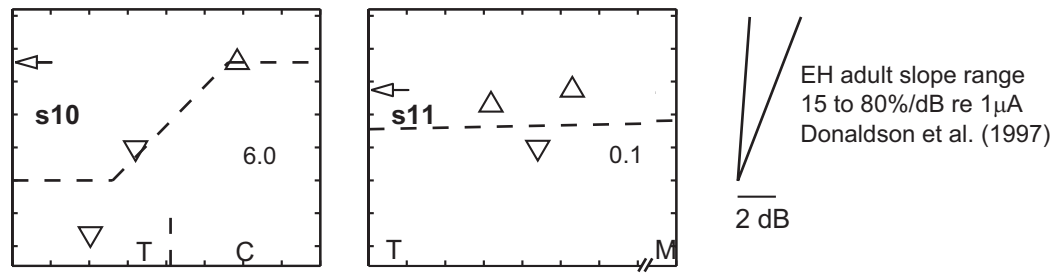

Signal Level ( $\mathrm{dB}$ re $1 \mu \mathrm{A})$

Fig. 1. Psychometric functions for detection from seven subjects, compared with clinical mapping parameters, and with published slope data from EH adults. Signal level is the average current-pulse amplitude in each current-pulse train (see Methods). Asymptotic performance is indicated by arrows on the ordinate. Downward triangles, diamonds, circles, and upward triangles indicate $p(C)_{\max }$, with $95 \%$ confidence intervals that include only chance, include both chance and asymptote, are contained entirely within the interval from chance to asymptote, or include only the asymptote, respectively. The three-line fit is plotted in dashed lines. The value of the slope is indicated on the right of each panel in units of percent correct per dB re $1 \mu \mathrm{A}$. The PFD threshold is shown by the vertical dashed line on the abscissa. To plot data on a common scale across subjects, for $\mathrm{s} 11$, the chance and asymptotic segments of the three-line fit and the PFD threshold $(27 \mathrm{~dB})$ are not shown. Similarly, for s9, the three-line-fit chance-segment is not shown. For s5 and s8, PFD data were obtained twice in two separate visits; 1 st measurement data are shown in gray, 2nd measurement in black. Clinical mapping parameters threshold (T) and clinical comfort level (C: Cochlear; $\mathrm{M}: \mathrm{AB}$ ) are indicated on the abscissa. The pair of lines in the bottom right depicts the range of EH adult slopes measured by Donaldson et al. (1997). These adult data are shown independent of threshold and plotted on the same scale as the child data.

$65 \mathrm{~dB}$ SPL acoustic tone bursts. All 11 subjects reached criterion in the first visit.

The number of trials required by each subject to reach training criterion is listed in the second column of Table 2. The median number of trials required to reach criterion was 14 , and 10 of the 11 children required 26 or fewer trials. Six children reached criterion within the minimum possible number of trials, given the particular random sequence of signal and no-signal trials presented. For example, correct responses occurred on all eight trials (four signal and four no-signal) presented to s9. The probability of reaching criterion because of guessing in this case would be less than $0.5 \%$. Overall, the EH children tended to reach training criterion more quickly than has been reported for younger NH infants, who typically take approximately 22 trials to reach criterion (Werner 1995).

Subject s2, who took 63 trials to reach criterion, has malformed cochleae with narrow internal auditory canals. Preimplant imaging revealed no visible auditory nerve. Thus, it may be that this child was receiving limited stimulation from the implant. Subject s7 did not reach training criterion but only completed one visit; her parent reported that the child was not feeling well at that time. Of note, both of these subjects often responded to signal presentations only after the trial had ended.

The number of trials required to reach criterion was not correlated with age $(r=0.17 ; p=0.61)$ or the duration of $\mathrm{EH}$ experience $(r=0.11 ; p=0.75)$. These correlations did not change if subject s2 was excluded.

\section{Characteristics of PFD}

The individual PFD are plotted in Figure 1. Complete hit and false alarm data are provided in the fourth column of Table
2. Correlation analyses were used to determine whether detection accuracy increased with stimulus level in the expected manner. As listed in the fifth column of Table 2, the correlation coefficient relating $p(C)_{\max }$ and stimulus level ranged from 0.83 to $1.00(p<0.000001)$ for all of the EH children except s11. Thus, children's detection improved with increasing signal level in the expected fashion, supporting the idea that OPP is measuring sensitivity to stimulation. These functions can be further described in terms of their upper asymptote, slope, and threshold.

Asymptote • The upper asymptote of the psychometric function approaches $100 \%$ for a perfectly attentive listener. However, because children are typically not perfectly attentive and because previous research has suggested that $\mathrm{EH}$ infants may not be as attentive to sound as are NH infants (Houston et al. 2003a), it was of interest to estimate the upper asymptote of the EH children's PFD. The upper asymptote was generally taken as the performance level at the highest signal level tested. Because it is not certain that children would not have performed better at a higher signal level, these estimates are conservative.

The estimated upper asymptotes are indicated by arrows in Figure 1 and listed in the sixth column of Table 2. Two values are shown for s5 and s8 because PFD data were collected twice in two separate visits. Across children, asymptotic performance ranged from 77 to $97 \%$, averaging $86 \%$. The $95 \%$ lower confidence interval for the asymptotic data point is also listed in the sixth column of Table 2 in parentheses. All subjects' 95\% lower confidence interval was greater than $50 \%$ (chance performance). The observed mean and range of asymptotic detection performance for 
EH children are comparable with those of NH 6 to 9 mo old infants reported by Bargones et al. (1995; $M=88 \%$; SD = $6 \% ; N=10)$ and those of NH 3 to $5 \mathrm{yr}$ old children tested in a standard OPP by Allen and Wightman (1994; $M=$ $90 \%$, SD not reported; $N=18$ ). Similar asymptotic detection performance suggests that EH children are as attentive to sound as $\mathrm{NH}$ children. Interestingly, there was a positive but nonsignificant correlation between asymptotic $p(C)_{\max }$ and both age ( $r=0.52 ; p=0.19$, two-tailed test) and the duration of EH experience ( $r=0.61 ; p=0.11$, two-tailed test). Slope - It was desirable to accurately estimate the PFD slope and base the estimate on all of the data collected. Assuming that PFD data follow a sigmoidal function, linear regression will necessarily underestimate the slope if any asymptotic point(s) are included. Slope, therefore, was estimated using a weighted average procedure that inversely weighted the slope of each piecewise linear section by the distance of the section from the threshold performance. ${ }^{\ddagger}$

The PFD was described by three line-segments such that the first and third segments had zero slope and described the lower and upper asymptotes. The upper asymptote was taken as the highest $p(C)_{\max }$ obtained. The lower asymptote was taken as chance performance. The middle segment was forced to pass through one point. The $95 \%$ confidence intervals for $p(C)_{\max }$ were used to determine this intersect point, which was defined as the mean of data point(s) whose 95\% confidence intervals included neither the upper nor lower asymptotes (i.e., points along the slope). In one case, s3, the mean of the points overlapping with both asymptotes was taken as the estimate of the intersect point. If no such point(s) were available, the intersect point was defined as the mean of the highest level point with confidence interval including chance and the lowest level point with confidence interval including the upper asymptote. If the highest lower asymptote point was less than chance, it was set to chance. The slope of the middle segment was given by $S$, defined as follows:

$$
S=\frac{\sum_{k=1}^{N-1} \frac{s_{k} \mathrm{n}_{k}}{d_{k}}}{\sum_{k=1}^{N-1} \frac{\mathrm{n}_{k}}{d_{k}}}
$$

where $N$ was the number of signal levels; $s_{k}=\left[p(C)_{\max }(k+\right.$ 1) $\left.-p(C)_{\max }(k)\right] /[\operatorname{level}(k+1)-$ level $(k)]$, the slope of the $k$-th section; $\mathrm{n}_{k}$ was the mean number of signal trials for the $k$-th section; and $d_{k}=\left[p(C)_{\max }(k)+p(C)_{\max }(k+1)\right] / 2-p_{T}$, the difference in $p(C)_{\max }$ between the midpoint of the $k$-th section and threshold performance $p_{\mathrm{T}}$, defined as the mean of the upper

\footnotetext{
\#Alternative methods were considered to calculate slope, including probit analysis (Finney 1977). However, because of few data points, when data were fitted with either normal or logistic cumulative distribution functions using least squares, error surfaces tended to lack a single well defined minimum along the slope dimension, suggesting unstable fits. The threeline fitting procedure of Bargones et al. (1995) was also considered. Compared with the proposed weighted average piecewise method, $R^{2}$ produced by the Bargones et al. method was lower in five cases, equivalent in two cases, and better in a single case.
}

asymptote and chance. Based on the definition of $S$, each piecewise section was inversely weighted by the distance of the section from threshold performance. Consequently, more weight was given to sections on the slope of the PFD and less weight to asymptotic- or chance-performance sections.

PFD three-line fits and data points are shown in Figure 1. Points not different from chance are indicated by downward triangles, points not different from asymptote are indicated by upward triangles, points not different from either chance or asymptote are indicated by diamonds, and points along the slope are indicated by circles. Calculated slope values are indicated in each panel (e.g., $6.3 \% / \mathrm{dB}$ for s3) and also given in the seventh column Table 2. $R^{2}$ was computed as a measure of goodness-of-fit for the three-line fits and is given in the eight column of Table 2 . In eight of nine functions, $R^{2}$ exceeded 0.77 . The exception occurred for s11 whose data were highly nonmonotonic.

The slope in EH might be expected to be steeper than that in $\mathrm{NH}$, because the dynamic range of $\mathrm{EH}$ is smaller than that of acoustic hearing. Slopes, however, were highly variable across these eight functions, ranging from 2 to $31 \% / \mathrm{dB}$. The distribution of slopes seemed bimodal. Some were comparable with NH children (1-6\%; Allen \& Wightman 1994; Bargones et al. 1995), and some were much steeper and comparable with the range of EH adult slopes measured by Donaldson et al. (1997) of 15 to $80 \% / \mathrm{dB}$ re $1 \mu \mathrm{A} .^{\S}$ To allow EH child-adult slope comparisons, the EH adult slope range measured by Donaldson et al. (1997) is plotted in the bottom right of Figure 1 and displayed on the same scale as the child data. Although these data provide a first estimate of EH infant and toddler PFD slopes, they should be viewed with caution given the limited numbers of levels they are based on $(\leq 6)$ and the number of trials obtained $(\leq 138)$.

PFD threshold $\bullet$ Clinical mapping parameters of threshold ( $T$ level) and comfort level (C level for Cochlear devices or $\mathrm{M}$ level for AB devices) are shown in relation to the PFD in Figure 1. For each panel, parameters are indicated at the location of the current-pulse amplitude measured using the implant-in-a-box when the clinical software was used to generate pulses with amplitude equal to the particular parameter. The PFD threshold is indicated by vertical lines on the level axis. PFD threshold was defined as the midpoint of the measured PFD.

For most subjects, the threshold of the PFD was between clinical threshold and comfort levels. For subjects s3 and s9, the threshold of the PFD was slightly below clinical threshold. Comfort levels were above the range of the PFD in all cases. Thus, in general, the PFD thresholds were consistent with the clinical parameters.

Reliability $\bullet$ The repeated PFD for subjects s5 and s8 are shown in black in Figure 1 and initial measurements are shown in gray. Repeated PFD parameters are listed in the second row of the entry for each subject in Table 2. The second PFD was obtained 41 and 14 days after the first PFD for s5 and s8, respectively. An adjustment in the levels tested was made in

\footnotetext{
${ }^{\$}$ PFD slope data from Donaldson et al. (1997) were compared with slopes obtained in this study by converting $\log \left(d^{\prime}\right)$ to $p(C)_{\max }$. A conversion factor of $50.6 \% p(C)_{\max } / \log \left(d^{\prime}\right)$ was used, which equals the slope of the function relating $p(C)_{\max }$ to $\log \left(d^{\prime}\right)$ in a two-interval task (cf., Macmillan \& Creelman, 2005), at the point where $d^{\prime}=1$ (i.e., threshold); $p(C)_{\max }$ equals $76 \%$ at this point. A slope range of 0.3 to $1.58 \log \left(d^{\prime}\right) / \mathrm{dB}$ re $1 \mu \mathrm{A}$ reported by Donaldson et al. (1997) was thus converted to a range of 15.2 to $79.9 \% / \mathrm{dB}$ re $1 \mu \mathrm{A}$.
} 


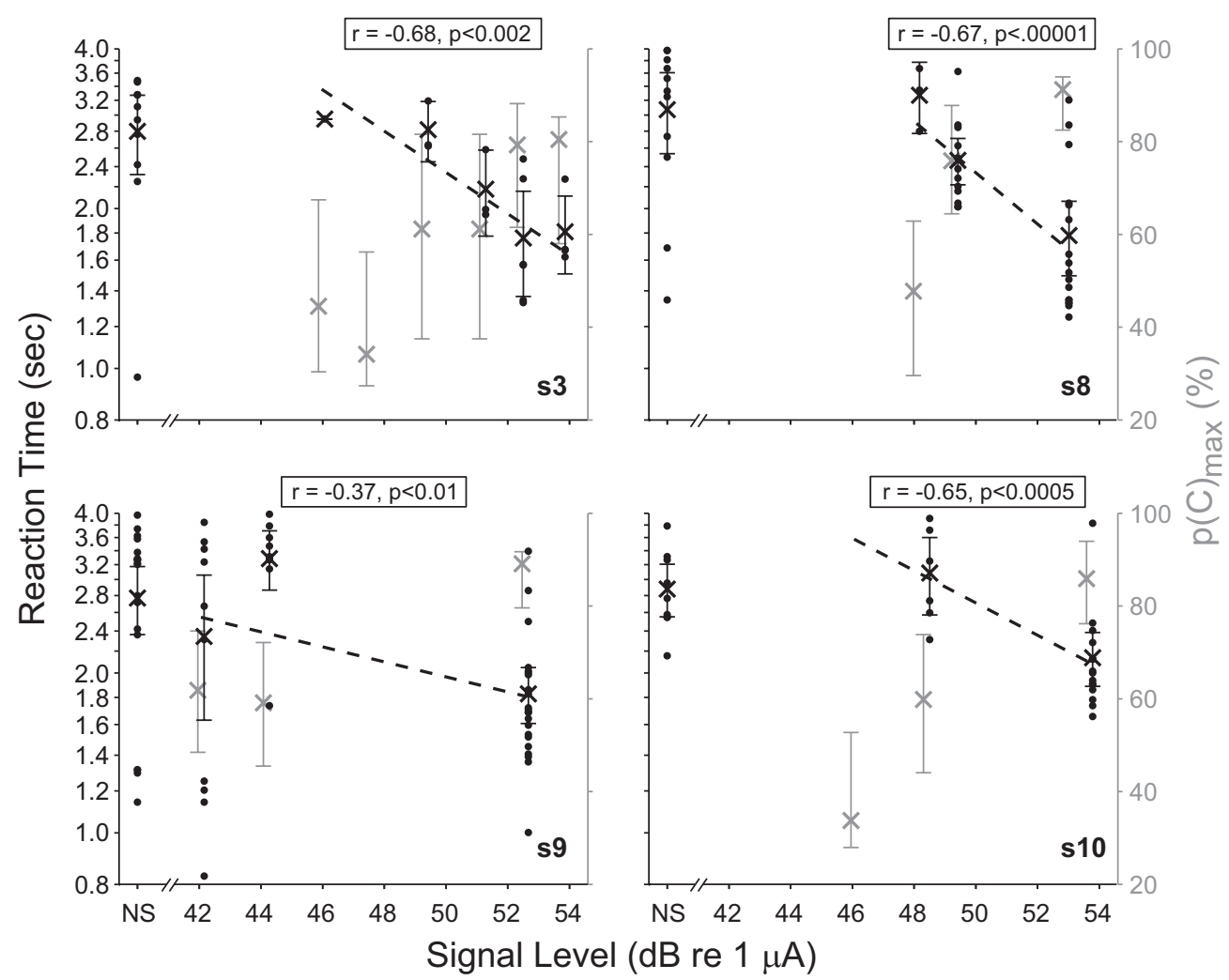

Fig. 2. In four subjects, reaction time decreased significantly with signal level, whereas detection accuracy increased. RT is shown on the left ordinate (in black), and $p\left(C_{\max }\right.$ on the right ordinate (in gray). Black dots indicate RT obtained on individual trials at each signal level, including no-signal "NS" trials; $x^{\prime}$ 's show mean RT; dashed lines show linear least-squares fits. The correlation coefficient $r$ between the logarithm of RT and signal level, and the significance of a negative relationship $p$, are indicated. Error bars show 95\% confidence intervals. Only second measurement data are plotted for s8. All RTs exceeded 0.8 sec. For clarity, RT and $p(C)_{\max }$ data have been slightly offset, by $\pm 0.1 \mathrm{~dB}$ of the actual signal level.

the second session for s8 to better define the PFD range. The same levels were tested in the two sessions for s5. For both subjects, detection performance was quite similar in the two sessions. For s5, the two thresholds were nearly identical. The PFD asymptote and slope were a little lower on retest, but this subject's PFD slope remained the steepest of those measured. For s8, threshold decreased by a dB or two, the upper asymptote increased slightly, and the PFD slope was steeper. The differences in 88 's PFD parameters may have more to do with the placement of the test levels than with the reliability of OPP; $p(C)_{\max }$ was only slightly better in the second session for the levels that were tested in both sessions. Thus, OPP yielded generally consistent results for these two subjects.

\section{Reaction Time}

The relationship between RT and stimulus level was examined to see whether RT provides information about the effects of stimulus level. In five of nine cases (four of seven subjects), RT decreased with stimulus level. Slope was computed in terms of the regression line relating the logarithm of RT to the logarithm of stimulus level, or $\log _{10}(\mathrm{RT})=m \log _{10}$ (current level in $\mu \mathrm{A})+b$. Slope $m$ is given in the final column of Table 2. A significant negative slope was observed in the four cases plotted in Figure 2. Both RT and $p(C)_{\max }$ are shown, RT by the black dots, mean RT by black "x," and $p(C)_{\max }$ by gray x. Error bars show the $95 \%$ confidence intervals for both data sets. The RT for false alarms is plotted at "NS", for no-signal trial, on the abscissa. The correlation coefficient $r$ for the relation between the logarithm of RT and stimulus level, and the associated one-tailed $p$ value, are indicated in each panel. For each case shown in Figure 2, mean RT for false alarms on no-signal presentations was significantly greater than it was for hits on signals presented at the highest level (one-tailed $t$-tests: $\mathrm{s} 3, p<$ $0.02 ; \mathrm{s} 8, p<0.0001 ; \mathrm{s} 9, p<0.0005 ; \mathrm{s} 10, p<0.001)$. No significant negative RT-versus-signal-level relationship was observed in three subjects ( $p>0.4$ : $\mathrm{s} 4$, $\mathrm{s} 5$ both measurements, $\mathrm{s} 11$, data not shown), although in one case, the mean RT from false alarms significantly exceeded the mean RT from hits on signals at the highest level (s5 2nd measurement, one-tailed $t$-test; $p<0.02$ ).

Overall, these data suggest that RT can reflect effects of stimulus level in EH children, although additional work is clearly required to allow such information to be reliably obtained from many children.

\section{DISCUSSION}

The results of this study indicate that OPP is a feasible method for obtaining psychophysical information about detection from infants and toddlers who use CIs. Eleven of 12 young EH children, of widely varying age, were successfully tested with OPP. OPP is based on the observation that auditory behavioral responses occur in even young $\mathrm{NH}$ infants, but that the responses change as infants develop (Northern \& Downs 2001). This feature of OPP has permitted testing of NH infants as young as 2 wk old (e.g., Werner \& Gillenwater 1990). It 
seems likely that auditory behavioral responses will also change as an EH infant's hearing experience increases. Consistent with that idea is the anecdotal observation that whereas some EH children in this study made head turns in response to sound, several made other responses, such as making a "listening" or "concentrating" face, making darting eye movements, or pointing to the reinforcer. Thus, the features that make OPP successful with very young infants may make it successful with older infants or toddlers who may just be starting to hear.

EH children's estimated asymptotic detection performance was $86 \%$ correct on average, comparable with $\mathrm{NH}$ infant's and children's detection of clearly audible tones (e.g., Allen \& Wightman 1994; Bargones et al. 1995). Under the assumption that asymptotic performance is an indication of attentiveness, these results suggest that $\mathrm{EH}$ children attend to sound as often as $\mathrm{NH}$ children do. In contrast, the results of Houston et al. (2003a) using preferential looking suggest that infant and toddler CI recipients with 6 mo or less of $\mathrm{EH}$ experience may not be particularly attentive to sound. In this procedure, looking at a visual pattern results in the presentation of sound as long as the infant maintains fixation on "sound" trials, whereas no sound follows from fixations on the pattern on "silent" trials. Houston et al. found that although NH 6 mo olds fixated significantly longer during sound than silent trials, EH toddlers with 6 mo of EH experience did not. Seven of the 11 children who participated in the current study had 6 or fewer months of EH experience, and the upper asymptote of their PFD was no different from that of the infants with longer EH experience. There are obvious differences between preferential looking and OPP that could account for the apparently contradictory findings. One possibility is that if infant and toddler CI recipients are less motivated by sound than $\mathrm{NH}$ children, they may not work as hard to hear a sound as $\mathrm{NH}$ children. In OPP, the reinforcer, as well as praise and encouragement from the parent and assistant, may motivate the child to respond to the sound, even if the sound itself is not interesting to the child (Trehub et al. 1981).

Based on our preliminary findings, the slope of young EH children's PFD seems more shallow than that of EH adults (Donaldson et al. 1997). The slope of the PFD may provide information about mechanisms underlying the detection process. For example, assuming that detection depends on the collective response of numerous spiral ganglion cells, each with a different sensitivity, then the slope would decrease with increases in the number of cells (cf., Watson et al. 1972). If the finding that EH children generally have shallower PFD slopes than $\mathrm{EH}$ adults is borne out by further research, it might suggest that $\mathrm{EH}$ children have more spiral ganglion neurons than $\mathrm{EH}$ adults do. Of course, inattentiveness or additive noise in the detection process would also be expected to decrease the PFD slope (Watson et al. 1972) and might well contribute to the apparent difference between EH children and adults in PFD slope.

Methodological differences between the child and adult studies, however, complicate the interpretation of the apparent age differences in PFD slope. On the one hand, the EH child-adult difference in slope may be greater than suggested. Donaldson et al. (1997) measured the PFD using $200 \mu$ s wide pulses, whereas the widest pulse width used in this study was $25 \mu \mathrm{s}$. Donaldson and Viemeister (Reference Note 1) showed that the PFD slope increased by 37 to $330 \%$ in five of seven $\mathrm{EH}$ adults as pulse width decreased from 200 to $30 \mu$ s. Thus, the EH adult slopes reported by Donaldson et al. (1997) may actually be steeper if pulse widths similar to those used in this study were presented. On the other hand, bipolar stimulation was used by Donaldson et al., whereas monopolar stimulation was used in this study. Monopolar stimulation produces a larger spatial extent of cochlear activation than bipolar (e.g., Kral et al. 1998; van den Honert \& Stypulkowski 1987), and likely recruits more spiral ganglion fibers with increases in stimulus level (e.g., Miller et al. 2003). Human psychophysical studies also indicate that monopolar stimulation results in a broader cochlear activation than does bipolar stimulation (e.g., Bierer 2007; Stickney et al. 2006). The slope of the PFD may consequently be shallower under monopolar than under bipolar stimulation, assuming that all other variables, including pulse width, are fixed. Further testing is required to clarify the effects of stimulation mode on PFD slope. Finally, the pulse rates used in this study were higher than the pulse rates used by Donaldson et al. How the difference in pulse rate would influence PFD slope has not been established.

The fact that RT decreased significantly with stimulus level in four of seven subjects for whom PFD were obtained is encouraging. Although RT was a less reliable measure of hearing than detection accuracy, it may prove useful in the study of loudness growth in EH infants and toddlers. This conclusion, of course, is based on few data. Methodological changes aimed specifically at establishing the relationship between RT and stimulus level in a larger number of subjects are required to definitively establish the use of RT as a measure of EH in young children. Anecdotal observations suggest that a significant RT effect was more frequently seen in children who mainly produced head-turn responses. Further research is required to determine whether the conditioned head turn procedure is particularly effective for measuring RT in young EH children. Leibold and Werner (2002) successfully used a conditioned head turn procedure to measure RT in NH 6 to 9 mo old infants.

One limitation of this study is the between-subject variability in the data obtained. Some between-subject variability in the performance of $\mathrm{EH}$ children is expected, based on the observed variability in EH adults' performance. The small sample size and wide age range of the subjects undoubtedly contribute, as well. However, another important contributor to variability is the small number of trials obtained. One solution to this problem would be to pool data across visits (e.g., Bargones et al. 1995). The similarity in the data obtained on repeated testing for two children in this study suggests that pooling data across visits would be a legitimate practice.

The stimulation paradigm used in this study may prove useful in future studies. LSES was developed to present controlled stimuli to a single electrode, without creating an unusual acoustic environment for the EH child. In LSES, a child could hear ambient sound on most other electrodes in a manner similar to his or her usual map. During sessions, children were cooperative and seemed to enjoy playing the listening game while being able to hear sounds from the reinforcer, the assistant, and their parent. Using LSES, some subjects' attention could be maintained for four, 10 to $20 \mathrm{~min}$ blocks in each visit, and at least 100 trials were collected from four subjects. This number of trials was never completed during pilot testing of subjects using direct single-electrode stimulation in which all other electrodes were turned off and there was no acoustic processing. In LSES, audio stimuli were presented directly to a sound processor that was programmed 
using standard clinical software. Similar methods may be beneficial in clinic or lab environments if programmable software and hardware that directly controls a recipient's implant is unavailable or when concurrent acoustic processing is desired. Given the fact that reinforcement itself produces lower thresholds in NH children (Trehub et al. 1981), the more usual auditory environment of LSES could provide more salient reinforcement for the child, which in turn, could contribute to lower and more accurate behavioral threshold levels in comparison with direct single-electrode stimulation.

\section{CONCLUSIONS}

We draw four conclusions from this study. First, OPP seems feasible for obtaining psychophysical detection data from EH infants and toddlers; thresholds obtained were comparable with clinical results. Second, auditory attention in EH infants and toddlers, as estimated by asymptotic-detection performance, seems comparable with levels reported in NH children. Third, the slope of EH children's PFD may be shallower than that of EH adults. Finally, methods like LSES, in which easy-togenerate signals are applied to the auxiliary input of a CI speech processor, may facilitate psychophysical testing of child and adult $\mathrm{CI}$ recipients in clinical and research settings.

\section{ACKNOWLEDGMENTS}

The authors thank all of the families for their dedicated participation. Gail Donaldson, Brenda Ryals, and three anonymous reviewers provided numerous helpful comments that substantially strengthened this article. Bas van Dijk and Colin Irwin at Cochlear Corporation and Leo Litvak at Advanced Bionics Corporation provided CI related assistance. $\mathrm{Xu}$ Yang and Nicole Paolone assisted in data collection. The CI team at Seattle Children's facilitated subject recruitment. Staff in the Infant Hearing Lab assisted in scheduling subjects. Programming and technical assistance was provided by Brandon Warren, Kelvin Wu, and Tom Lewis. Steve Colburn, Ward Drennen, Chris Stecker, Tina Grieco, Jong Ho Won, Barbara Cone-Wesson, and staff at the Listen and Talk school provided helpful comments on this work.

This work was supported by NIH T32 DC000018, T32 DC000033, F32 DC008238, the National Organization for Hearing Research Foundation, and a gift from the estate of Verne J. Wilkins. NIH P30 DC004661 and P30 HD02274 also facilitated this study.

Address for correspondence: Vasant K. Dasika, Virginia Merrill Bloedel Hearing Research Center, University of Washington, Box 357923, Seattle, WA 98195. E-mail: vasant.dasika@gmail.com

Received October 13, 2007; accepted October 13, 2008.

\section{REFERENCES}

Allen, P., \& Wightman, F. (1994). Psychometric functions for children's detection of tones in noise. $J$ Speech Hear Res, 37, 205-215.

Bargones, J. Y., Werner, L. A., \& Marean, G. C. (1995). Infant psychometric functions for detection: mechanisms of immature sensitivity. $J$ Acoust Soc Am, 98, 99-111.

Barker, B. A., \& Tomblin, J. B. (2004). Bimodal speech perception in infant hearing aid and cochlear implant users. Arch Otolaryngol Head Neck Surg, 130, 582-586.

Bierer, J. A. (2007). Threshold and channel interaction in cochlear implant users: evaluation of the tripolar electrode configuration. $J$ Acoust Soc Am, 121, 1642-1653.

Carlyon, R. P., Buus, S., \& Florentine, M. (1990). Temporal integration of trains of tone pulses by normal and by cochlearly impaired listeners. $J$ Acoust Soc Am, 87, 260-268.
Donaldson, G. S., Viemeister, N. F., \& Nelson, D. A. (1997). Psychometric functions and temporal integration in electric hearing. $J$ Acoust Soc Am, 101, 3706-3721.

Eisenberg, L. S., Johnson, K. C., \& Martinez, A. S. (2005). Clinical assessment of speech perception for infants and toddlers. [Article from Audiology Online web site]. 2005, September 12. Retrieved July 20, 2007 from http://www.audiologyonline.com/articles/article_detail.asp?article_id $=1443$.

Eisenberg, L. S., Johnson, K. C., Martinez, A. S., et al. (2006). Speech recognition at 1 -year follow-up in the childhood development after cochlear implantation study: methods and preliminary findings. Audiol Neurootol, 11, 259-268.

Eisenberg, L. S., Martinez, A. S., \& Boothroyd, A. (2007). Assessing auditory capabilities in young children. Int J Pediatr Otorhinolaryngol, $71,1339-1350$.

Epstein, M., \& Florentine, M. (2006). Reaction time to 1- and 4-khz tones as a function of sensation level in listeners with normal hearing. Ear Hear, 27, 424-429.

Finney, D. J. (1977). Probit Analysis. Cambridge: Cambridge University Press.

Gfeller, K., Witt, S. A., Spencer, L. J., et al. (1999). Musical involvement and enjoyment of children who use cochlear implants. Volta Rev, 100, 213-233.

Grieco-Calub, T. M., Litovsky, R. Y., \& Werner, L. A. (2008). Using the observer-based psychophysical procedure to assess localization acuity in toddlers who use bilateral cochlear implants. Otol Neurotol, 29, 235-239.

Heil, P., Neubauer, H., Tiefenau, A., et al. (2006). Comparison of absolute thresholds derived from an adaptive forced-choice procedure and from reaction probabilities and reaction times in a simple reaction time paradigm. J Assoc Res Otolaryngol, 7, 279-298.

Horn, D. L., Pisoni, D. B., \& Miyamoto, R. T. (2006). Divergence of fine and gross motor skills in prelingually deaf children: implications for cochlear implantation. Laryngoscope, 116, 1500-1506.

Houston, D. M., Pisoni, D. B., Kirk, K. I., et al. (2003a). Speech perception skills of deaf infants following cochlear implantation: a first report. Int $J$ Pediatr Otorhinolaryngol, 67, 479-495.

Houston, D. M., Ying, E. A., Pisoni, D. B., et al. (2003b). Development of preword-learning skills in infants with cochlear implants. Volta Rev, 103, 303-326.

Humes, L. E., \& Ahlstrom, J. B. (1984). Relation between reaction time and loudness. J Speech Hear Res, 27, 306-310.

Kral, A., Hartmann, R., Mortazavi, D., et al. (1998). Spatial resolution of cochlear implants: the electrical field and excitation of auditory afferents. Hear Res, 121, 11-28.

Leibold, L. J., \& Werner, L. A. (2002). Relationship between intensity and reaction time in normal-hearing infants and adults. Ear Hear, 23, 92-97.

Luce, R. D. (1991). Response Times: Their Role in Inferring Elementary Mental Organization. New York: Oxford University Press.

Macmillan, N. A., \& Creelman, C. D. (2005). Detection Theory: A User's Guide. Hillsdale, NJ: Lawrence Erlbaum Associates.

Marean, G. C., Werner, L. A., \& Kuhl, P. K. (1992). Vowel categorization by very young infants. Dev Psychol, 28, 396-405.

Miller, C. A., Abbas, P. J., Nourski, K. V., et al. (2003). Electrode configuration influences action potential initiation site and ensemble stochastic response properties. Hear Res, 175, 200-214.

Miyamoto, R. T., Houston, D. M., \& Bergeson, T. (2005). Cochlear implantation in deaf infants. Laryngoscope, 115, 1376-1380.

Mukari, S. Z., Ling, L. N., \& Ghani, H. A. (2007). Educational performance of pediatric cochlear implant recipients in mainstream classes. Int $J$ Pediatr Otorhinolaryngol, 71, 231-240.

Nakata, T., Trehub, S. E., Mitani, C., et al. (2005). Music recognition by japanese children with cochlear implants. J Physiol Anthropol Appl Human Sci, 24, 29-32.

Northern, J. L., \& Downs, M.P. (2001). Hearing in Children. Philadelphia: Lippincott Williams \& Wilkins.

Olsho, L., Koch, E., Halpin, C., et al. (1987). An observer-based psychoacoustic procedure for use with young infants. Dev Psychol, 23, $627-640$.

Olszewski, C., Gfeller, K., Froman, R., et al. (2006). Familiar melody recognition by children and adults using cochlear implants and normal hearing children cochlear implants. Cochlear Implants Int, 6, 123-140.

Pfingst, B. E. (1984). Operating ranges and intensity psychophysics for cochlear implants. Implications for speech processing strategies. Arch Otolaryngol, 110, 140-144. 
Pfingst, B. E., Hienz, R., \& Miller, J. (1975). Reaction-time procedure for measurement of hearing. II. Threshold functions. J Acoust Soc Am, 57, 431-436.

Schneider, B. A., Trehub, S. E., Morrongiello, B. A., et al. (1989). Developmental changes in masked thresholds. J Acoust Soc Am, 86, 1733-1742.

Stickney, G. S., Loizou, P. C., Mishra, L. N., et al. (2006). Effects of electrode design and configuration on channel interactions. Hear Res, $211,33-45$.

Stordahl, J. (2002). Song recognition and appraisal: a comparison of children who use cochlear implants and normally hearing children. J Music Ther, 39, 2-19.

Trehub, S. E., Schneider, B. A., \& Endman, M. (1980). Developmental changes in infants' sensitivity to octave-band noises. $J$ Exp Child Psychol, 29, 282-293.

Trehub, S. E., Schneider, B. A., \& Bull, D. (1981). Effect of reinforcement on infants' performance in an auditory detection task. Dev Psychol, 17, 872-877.

Uziel, A. S., Sillon, M., Vieu, A., et al. (2007). Ten-year follow-up of a consecutive series of children with multichannel cochlear implants. Otol Neurotol, 28, 615-628.

van den Honert, C., \& Stypulkowski, P. H. (1987). Single fiber mapping of spatial excitation patterns in the electrically stimulated auditory nerve. Hear Res, 29, 195-206.

Vongpaisal, T., Trehub, S. E., \& Schellenberg, E. G. (2006). Song recognition by children and adolescents with cochlear implants. J Speech Lang Hear Res, 49, 1091-1103.

Walloch, R. A., Fenwick, J. A., \& Boberg, R. (1980). A reaction-time analysis of electrocochlear stimulation in cats. Laryngoscope, 90, 861-866.
Watson, C. S., Franks, J. R., \& Hood, D. C. (1972). Detection of tones in the absence of external masking noise: I-Effects of signal intensity and signal frequency. $J$ Acoust Soc Am, 52, 633-643.

Werker, J. F., Polka, L., \& Pegg, J. E. (1997). The conditioned head turn procedure as a method for assessing infant speech perception. Early Development and Parenting, 6, 171-178.

Werner, L. A. (1995). Observer-Based Approaches to Human Infant Psychophysics. In R. Dooling, R. R. Fay, G. Klump, and W. Stebbins (Eds.). Methods in Comparative Psychoacoustics (pp, 135-146). Basel: Birkhäuser Verlag.

Werner, L. A., \& Boike, K. (2001). Infants' sensitivity to broadband noise. $J$ Acoust Soc Am, 109, 2103-2111.

Werner, L. A., \& Marean, G. C. (1991). Methods for estimating infant thresholds. $J$ Acoust Soc Am, 90, 1867-1875.

Werner, L. A., \& Gillenwater, J. M. (1990). Pure-tone sensitivity of 2- to 5-week-old infants. Infant Behav Dev, 13, 355-375.

\section{REFERENCE NOTES}

1. Donaldson, G. S., \& Viemeister, N. F. (2001). Psychometric functions for detection and temporal integration: Pulse-duration effects. Paper presented at the 2001 Conference on Implantable Auditory Prostheses, Asilomar, CA.

2. Johnson, K. C., Wygonski, J. J., \& Eisenberg, L. S. (2005). Choice reaction time (CRT) in assessing pediatric word and sentence recognition. Paper presented at the 10th Symposium on Cochlear Implant in Children, Dallas, TX. Abstract retrieved April 28, 2008 from http:// www4.utsouthwestern.edu/continuinged/ci2005/cochlear_abstracts.pdf. 